\title{
THE ENERGY DECAY AND ASYMPTOTICS FOR A CLASS OF SEMILINEAR WAVE EQUATIONS IN TWO SPACE DIMENSIONS
}

\author{
SOICHIRO KATAYAMA, DAISUKE MUROTANI, AND HIDEAKI SUNAGAWA
}

\begin{abstract}
We consider semilinear wave equations with small initial data in two space dimensions. For a class of wave equations with cubic nonlinearity, we show the global existence of small amplitude solutions, and give an asymptotic description of the solution as $t \rightarrow \infty$ uniformly in $x \in \mathbb{R}^{2}$. In particular, our result implies the decay of the energy when the nonlinearity is dissipative.
\end{abstract}

\section{INTRODUCTION}

We consider the Cauchy problem for the following type of semilinear wave equations with small data:

$$
\begin{array}{ll}
\square u=F(\partial u), & (t, x) \in(0, \infty) \times \mathbb{R}^{2}, \\
u(0, x)=\varepsilon f(x), \partial_{t} u(0, x)=\varepsilon g(x), & x \in \mathbb{R}^{2},
\end{array}
$$

where $u$ is a real-valued unknown function of $(t, x) \in[0, \infty) \times \mathbb{R}^{2}, \square=\partial_{t}^{2}-\Delta=$ $\partial_{t}^{2}-\left(\partial_{1}^{2}+\partial_{2}^{2}\right)$, and $\partial u=\left(\partial_{0} u, \partial_{1} u, \partial_{2} u\right)$ with the notation $\partial_{0}=\partial_{t}=\partial / \partial t$, and $\partial_{j}=\partial / \partial x_{j}(j=1,2)$. We suppose that $f, g \in C_{0}^{\infty}\left(\mathbb{R}^{2} ; \mathbb{R}\right) . \varepsilon$ is a small positive parameter. We assume that the nonlinear term $F=F(q)$ is a $C^{\infty}$ function of $q=\left(q_{0}, q_{1}, q_{2}\right) \in \mathbb{R}^{3}$.

The local existence of classical solutions to (1.1)-(1.2) is well known, and we are interested in the sufficient condition for the global existence of the solutions, and also in the asymptotic behavior of global solutions. We say that the small data global existence (or SDGE) holds if for any $f, g \in C_{0}^{\infty}\left(\mathbb{R}^{2}\right)$ there is a positive constant $\varepsilon_{0}$ such that (1.1)-(1.2) possesses a global solution $u \in C^{\infty}\left([0, \infty) \times \mathbb{R}^{2}\right)$ for $0<\varepsilon \leq \varepsilon_{0}$. The case of cubic nonlinearity, that is to say $F(q)=O\left(|q|^{3}\right)$ near $q=0$, is critical in two space dimensions, because SDGE holds for some nonlinearity and fails for others. For example, SDGE does not hold for $F=\left(\partial_{t} u\right)^{3}$, however SDGE holds when $F$ is cubic and the null condition for cubic terms (we refer to it as the cubic null condition) is satisfied (see Godin [4]). To be more specific, we say that $F$ satisfies the cubic null condition if $F^{(c)}\left(-1, \omega_{1}, \omega_{2}\right)=0$ for any $\omega=\left(\omega_{1}, \omega_{2}\right) \in \mathbb{S}^{1}$, where $F^{(c)}$

1991 Mathematics Subject Classification. Primary 35L71; Secondary 35B40.

Key words and phrases. Nonlinear wave equations; asymptotic behavior; nonlinear dissipation; energy decay. 
denotes the cubic homogeneous part of $F$, that is,

$$
F^{(c)}(q)=\lim _{\lambda \rightarrow+0} \lambda^{-3} F(\lambda q) .
$$

The typical example satisfying the cubic null condition is

$$
F(\partial u)=\sum_{a=0}^{2} c_{a}\left(\partial_{a} u\right)\left(\left|\partial_{t} u\right|^{2}-\left|\nabla_{x} u\right|^{2}\right)
$$

with arbitrary constants $c_{a}$. The null condition was first introduced for systems of nonlinear wave equations with quadratic nonlinearity in three space dimensions as a sufficient condition to ensure SDGE (see Klainerman [14] and Christodoulou [3]; note that the case of quadratic nonlinearity is critical in three space dimensions). The terms satisfying the null condition form an important class of nonlinearity. We do not go into details, but the global existence under the null condition for the quasi-linear systems, even with quadratic nonlinearity, in two space dimensions is also studied by many authors (see [1], [2], [8], [10], and [11] for example).

Another important class of nonlinearity is the nonlinear dissipation. To explain the situation clearly, we consider the following equation in general space dimensions:

$$
\begin{array}{ll}
\square u=-\left|\partial_{t} u\right|^{p-1}\left(\partial_{t} u\right), & (t, x) \in(0, \infty) \times \mathbb{R}^{n}, \\
u(0, x)=\varphi(x), \partial_{t} u(0, x)=\psi(x), & x \in \mathbb{R}^{n},
\end{array}
$$

where $p>1$. It is known that there is a global solution $u \in C\left([0, \infty) ; H^{1}\left(\mathbb{R}^{n}\right)\right) \cap$ $C^{1}\left([0, \infty) ; L^{2}\left(\mathbb{R}^{n}\right)\right)$ for $(\varphi, \psi) \in X_{p}:=H^{2}\left(\mathbb{R}^{n}\right) \times\left(H^{1}\left(\mathbb{R}^{n}\right) \cap L^{2 p}\left(\mathbb{R}^{n}\right)\right)$ (see LionsStrauss [17] for instance). Here no smallness of the data is required. We define the energy norm by

$$
\|u(t)\|_{E}^{2}:=\frac{1}{2} \int_{\mathbb{R}^{n}}\left(\left|\partial_{t} u(t, x)\right|^{2}+\left|\nabla_{x} u(t, x)\right|^{2}\right) d x .
$$

Mochizuki-Motai [18] proved that if $n \geq 1$ and $1<p \leq 1+2 / n$, then the energy decays to zero, namely $\lim _{t \rightarrow \infty}\|u(t)\|_{E}=0$ for initial data belonging to a dense subset of $X_{p}$ (see also Todorova-Yordanov [21]). On the other hand, in the case of $n \geq 2$ and $p>1+2 /(n-1)$, it is also proved in [18] that the energy does not decay for a class of small initial data in $X_{p}$. To sum up, the result in [18] for $n=2$ can be read as follows: The energy decays for initial data in a dense subset of $X_{p}$ if $1<p \leq 2$, while the energy does not decay for small initial data if $p>3$. There is a gap in the conditions for $p$, and it is quite interesting to investigate what happens for (1.3) with $n=2$ and $p=3$ (or equivalently (1.1) with $F(\partial u)=-\left|\partial_{t} u\right|^{2} \partial_{t} u$ ).

The result above suggests that SDGE holds for the nonlinearity $F(\partial u)=$ $-\left|\partial_{t} u\right|^{2} \partial_{t} u$, though it does not satisfy the cubic null condition. Hence it is natural to expect that there is a sufficient condition for SDGE which is weaker than the cubic null condition and includes also the nonlinear dissipative terms. 
Agemi conjectured that the condition

$$
F^{(c)}\left(-1, \omega_{1}, \omega_{2}\right) \geq 0, \quad \omega=\left(\omega_{1}, \omega_{2}\right) \in \mathbb{S}^{1}
$$

implies SDGE. This conjecture was proved to be true by Hoshiga [9] and Kubo 15 independently. Moreover some asymptotic pointwise behavior of global solutions under (1.5) is obtained in [15] (see also Hayashi-NaumkinSunagawa [5] and Sunagawa [20] for related results on nonlinear Schrödinger equations and nonlinear Klein-Gordon equations, respectively). Let us restrict our attention to the case of the nonlinear dissipative term $F(\partial u)=-\left|\partial_{t} u\right|^{2} \partial_{t} u$. Then, it follows from [15] that

$$
|\partial u(t, x)| \leq C r^{-1 / 2}\left(\log \frac{r}{|r-t-1|+1}\right)^{-1 / 2}, \quad r \geq \frac{t}{2}+1
$$

with some positive constant $C$, where $r=|x|$. This estimate is improved in [19] as follows:

$$
|\partial u(t, x)| \leq C t^{-1 / 2} \min \left\{(\log t)^{-1 / 2}, \varepsilon(1+|r-t|)^{-\vartheta}\right\}
$$

for $(t, x) \in[2, \infty) \times \mathbb{R}^{2}$ with $0<\vartheta<1 / 2$. However this is still insufficient in order to say something about the decay of the energy.

In this paper we will further improve the pointwise estimate of $\partial u$ under the condition (1.5), and show that the energy decays to zero if we consider (1.1) with $F(\partial u)=-\left|\partial_{t} u\right|^{2} \partial_{t} u$ (or (1.3) with $n=2$ and $p=3$ in other words) for small $C_{0}^{\infty}$-data (in fact, complex-valued solutions will be considered).

\section{The MAIN RESUlt AND ITS APPLICATIONS}

2.1. Global existence and asymptotic pointwise behavior. In what follows, we consider the initial value problem (1.1)-(1.2) for complex-valued data with the nonlinearity

$$
F(\partial u)=\sum_{a, b, c=0}^{2} p_{a b c}\left(\partial_{a} u\right)\left(\partial_{b} u\right)\left(\overline{\partial_{c} u}\right)
$$

with complex constants $p_{a b c}$ in order that we can catch up two kinds of interesting nonlinearities $F(\partial u)=-\left|\partial_{t} u\right|^{2}\left(\partial_{t} u\right)$ and $F(\partial u)=i\left|\partial_{t} u\right|^{2}\left(\partial_{t} u\right)$ (see Subsections 2.2 and 2.3 below). Here and hereafter, $\bar{z}$ denotes the complex conjugate of $z \in \mathbb{C}$ and the symbol $i$ always stands for $\sqrt{-1}$. We also use the notation $\langle z\rangle=\sqrt{1+|z|^{2}}$. The following theorem is our main result.

Theorem 2.1. Assume that (2.1) is satisfied. We also assume

$$
\operatorname{Re} F(\hat{\omega}) \geq 0, \quad \hat{\omega}=\left(\omega_{0}, \omega\right) \in\{-1\} \times \mathbb{S}^{1} .
$$

Let $\mu$ be a sufficiently small positive constant satisfying $0<\mu<1 / 10$, say. Then, for any $f, g \in C_{0}^{\infty}\left(\mathbb{R}^{2} ; \mathbb{C}\right)$, there exists a positive constant $\varepsilon_{0}$ such that (1.1) -(1.2) admits a unique global classical solution $u \in C^{\infty}\left([0, \infty) \times \mathbb{R}^{2} ; \mathbb{C}\right)$ 
for any $\varepsilon \in\left(0, \varepsilon_{0}\right]$. Moreover, there is a function $P_{0}$ of $(\sigma, \omega) \in \mathbb{R} \times \mathbb{S}^{1}$, which satisfies

$$
\left|P_{0}(\sigma, \omega)\right| \leq C \varepsilon\langle\sigma\rangle^{\mu-1}, \quad(\sigma, \omega) \in \mathbb{R} \times \mathbb{S}^{1}
$$

with a positive constant $C$, such that

$$
\partial u(t, x)=\hat{\omega}(x) t^{-1 / 2} P\left(\log t,|x|-t,|x|^{-1} x\right)+O\left(\varepsilon t^{4 \mu-(3 / 2)}\langle t-|x|\rangle^{-3 \mu}\right)
$$

for any $(t, x) \in[1, \infty) \times \mathbb{R}^{2}$, where $\hat{\omega}(x)=\left(-1,|x|^{-1} x_{1},|x|^{-1} x_{2}\right)$ for $x=$ $\left(x_{1}, x_{2}\right) \in \mathbb{R}^{2}$, and $P=P(\tau, \sigma, \omega)$ is defined as a solution to

$$
\left\{\begin{array}{l}
\partial_{\tau} P(\tau, \sigma, \omega)=-(F(\hat{\omega}) / 2)|P(\tau, \sigma, \omega)|^{2} P(\tau, \sigma, \omega), \quad \tau>0, \\
P(0, \sigma, \omega)=P_{0}(\sigma, \omega)
\end{array}\right.
$$

with $\hat{\omega}=(-1, \omega)$. Here the constant $C$ is independent of $\varepsilon$.

Remark 2.1. (1) It is well known that if $f=g=0$ for $|x| \geq R$, then $u(t, x)=0$ for $|x| \geq t+R$ (see Hörmander [7] for instance). Hence $t$ is equivalent to $\langle t+|x|\rangle$ in $\operatorname{supp} u(t, \cdot)$ when $t \geq 1$.

(2) $P(\tau, \sigma, \omega)$ in the above theorem can be explicitly solved as

$$
P(\tau, \sigma, \omega)=P_{0}(\sigma, \omega) \frac{\exp (-i \Theta(\tau, \sigma, \omega))}{\sqrt{1+(\operatorname{Re} F(\hat{\omega}))\left|P_{0}(\sigma, \omega)\right|^{2} \tau}}
$$

with

$$
\Theta(\tau, \sigma, \omega)=\frac{1}{2}(\operatorname{Im} F(\hat{\omega})) \int_{0}^{\tau} \frac{\left|P_{0}(\sigma, \omega)\right|^{2}}{1+(\operatorname{Re} F(\hat{\omega}))\left|P_{0}(\sigma, \omega)\right|^{2} \tau^{\prime}} d \tau^{\prime} .
$$

From (2.6), we have

$$
|P(\tau, \sigma, \omega)| \leq \frac{\left|P_{0}(\sigma, \omega)\right|}{\sqrt{1+(\operatorname{Re} F(\hat{\omega}))\left|P_{0}(\sigma, \omega)\right|^{2} \tau}} .
$$

(3) We can add higher order nonlinear terms to (2.1), but the result becomes slightly weaker from the viewpoint of the estimate for the remainder term: Theorem 2.1 remains valid if we replace (2.1) by

$$
F(\partial u)=\sum_{a, b, c=0}^{2} p_{a b c}\left(\partial_{a} u\right)\left(\partial_{b} u\right)\left(\overline{\partial_{c} u}\right)+O\left(|\partial u|^{4}\right) \quad \text { near } \partial u=0,
$$

$F$ in (2.2) as well as in (2.5) by $F^{(c)}$, and $O\left(\varepsilon t^{4 \mu-(3 / 2)}\langle t-|x|\rangle^{-3 \mu}\right)$ in (2.4) by $O\left(\varepsilon t^{\mu-1}\langle t-|x|\rangle^{-1 / 2}\right)$.

We will prove Theorem 2.1 in Section 6 after some preparation given in Sections [3, 4 and 5. Compared to the method by Kubo [15], the most different point in our proof is the choice of the equation (2.5) for the asymptotics. Careful treatment of the factor $\langle t-|x|\rangle$ is also quite important in our improvement.

In the following subsections, we discuss what we can see from Theorem 2.1 particularly in the cases of $F(\partial u)=-\left|\partial_{t} u\right|^{2} \partial_{t} u$ (Subsection 2.2) and $F(\partial u)=$ $i\left|\partial_{t} u\right|^{2} \partial_{t} u$ (Subsection 2.3). 
2.2. The case of nonlinear dissipation: The decay of the energy. We focus on the case where the inequality in (2.2) is strict, i.e.,

$$
C_{0}:=\min _{\omega \in \mathbb{S}^{1}} \operatorname{Re} F(\hat{\omega})>0 .
$$

The typical example satisfying (2.9) is $F(\partial u)=-\left|\partial_{t} u\right|^{2}\left(\partial_{t} u\right)$. In this case, it follows from (2.3) and (2.8) that

$$
|P(\tau, \sigma, \omega)| \leq \min \left\{\frac{1}{\sqrt{C_{0} \tau}}, C \varepsilon\langle\sigma\rangle^{\mu-1}\right\} .
$$

Hence, by (2.4) we can find a positive constant $C$ such that

$$
|\partial u(t, x)| \leq C t^{-1 / 2} \min \left\{(\log t)^{-1 / 2}, \varepsilon\langle t-|x|\rangle^{\mu-1}\right\}
$$

for $(t, x) \in[2, \infty) \times \mathbb{R}^{2}$. This estimate says that $\partial u$ decays like $(t \log t)^{-1 / 2}$ along the line $l_{\sigma}:=\{(t, x) ;|x|-t=\sigma\}$ for each $\sigma \in \mathbb{R}$. On the other hand, for the solution $u_{0}$ to the free wave equation $\square u_{0}=0$ with $C_{0}^{\infty}$-data, it is known that $\partial u_{0}$ decays at the rate of $t^{-1 / 2}$ along $l_{\sigma}$. Hence (2.10) tells us that $\partial u$ has a gain of $(\log t)^{-1 / 2}$ in the pointwise decay compared to $\partial u_{0}$. Moreover, from (2.10) we obtain the following decay of the energy:

Corollary 2.2. Suppose that (2.1) and (2.9) are fulfilled, and let $0<\mu<$ $1 / 10$. Then for any $f, g \in C_{0}^{\infty}\left(\mathbb{R}^{2} ; \mathbb{C}\right)$, the global solution $u$ to (1.1) -(1.2) satisfies

$$
\|u(t)\|_{E}^{2} \leq C \varepsilon^{\frac{1}{1-\mu}}(\log t)^{-\frac{1-2 \mu}{2-2 \mu}}, \quad t \geq 2
$$

with some positive constant $C$, provided that $\varepsilon$ is sufficiently small.

The proof for this result will be given in Section 7 . This corollary says that the energy non-decay result of Mochizuki-Motai [18] fails for $n=2$ and $p=3$. It also suggests that the energy decay result in [18] holds also for $n=2$ and $2<p \leq 3$, but this is still an open problem (note that the energy decay for $n=2$ and $p=3$ established here is only for small $C_{0}^{\infty}$-data).

2.3. The case without dissipation: Logarithmic correction of the phase. We say that the global solution $u$ to (1.1)-(1.2) is asymptotically free (in the energy sense) if there is $\left(\varphi_{+}, \psi_{+}\right) \in \dot{H}^{1}\left(\mathbb{R}^{2}\right) \times L^{2}\left(\mathbb{R}^{2}\right)$ such that

$$
\lim _{t \rightarrow \infty}\left\|u(t)-u_{+}(t)\right\|_{E}=0 \text {, }
$$

where $u_{+}$is the solution to the free wave equation $\square u_{+}=0$ in the energy class with initial data $\left(u_{+}, \partial_{t} u_{+}\right)=\left(\varphi_{+}, \psi_{+}\right)$at $t=0$. Here $\dot{H}^{1}\left(\mathbb{R}^{2}\right)$ is the completion of $C_{0}^{\infty}\left(\mathbb{R}^{2}\right)$ with respect to the norm $\|\phi\|_{\dot{H}^{1}\left(\mathbb{R}^{2}\right)}=\|\nabla \phi\|_{L^{2}\left(\mathbb{R}^{2}\right)}$. It is proved in Katayama [13. (see also [12]) that $u$ is asymptotically free if and only if there is $U=U(\sigma, \omega) \in L^{2}\left(\mathbb{R} \times \mathbb{S}^{1}\right)$ such that

$$
\lim _{t \rightarrow \infty}\|\partial u(t, \cdot)-\hat{\omega}(\cdot) \widetilde{U}(t, \cdot)\|_{L^{2}\left(\mathbb{R}^{2}\right)}=0
$$

where $\hat{\omega}(x)=\left(-1, x_{1} /|x|, x_{2} /|x|\right)$ and $\widetilde{U}(t, x)=|x|^{-1 / 2} U(|x|-t, x /|x|)$. 
Now we consider the case of

$$
\operatorname{Re} F(\hat{\omega})=0, \quad \omega \in \mathbb{S}^{1}
$$

which is stronger than (2.2) but weaker than the cubic null condition. In this case, $P$ can be written as

$$
P(\tau, \sigma, \omega)=P_{0}(\sigma, \omega) \exp (-i \widetilde{\Theta}(\sigma, \omega) \tau)
$$

with $\widetilde{\Theta}(\sigma, \omega)=(\operatorname{Im} F(\hat{\omega}))\left|P_{0}(\sigma, \omega)\right|^{2} / 2(\in \mathbb{R})$. Hence we find from (2.4) that $\partial u$ decays at the same rate of $t^{-1 / 2}$ as the derivatives of the free solution $u_{0}$ along the line $l_{\sigma}$ for each $\sigma$. By (2.3), (2.4), and (2.12), we get

$$
\begin{aligned}
\|\partial u(t, \cdot)-\hat{\omega}(\cdot) \widetilde{P}(t, \cdot)\|_{L^{2}\left(\mathbb{R}^{2}\right)} & \leq C \varepsilon t^{2 \mu-(1 / 2)}\left\||\cdot|^{-1 / 2}\langle t-|\cdot|\rangle^{-\mu-(1 / 2)}\right\|_{L^{2}\left(\mathbb{R}^{2}\right)} \\
& \leq C \varepsilon t^{2 \mu-(1 / 2)} \rightarrow 0 \quad(t \rightarrow \infty),
\end{aligned}
$$

where $\widetilde{P}(t, x)=|x|^{-1 / 2} P(\log t,|x|-t, x /|x|)$. Moreover (2.3) and (2.12) lead to

$$
\lim _{t \rightarrow \infty}\|P(\log t, \cdot, \cdot)\|_{L^{2}\left((-\infty,-t) \times \mathbb{S}^{1}\right)}=0 .
$$

Therefore we find that $u$ is asymptotically free if and only if there is $U=$ $U(\sigma, \omega) \in L^{2}\left(\mathbb{R} \times \mathbb{S}^{1}\right)$ such that

$$
\lim _{\tau \rightarrow \infty}\|P(\tau, \cdot, \cdot)-U(\cdot, \cdot)\|_{L^{2}\left(\mathbb{R} \times \mathbb{S}^{1}\right)}=0 .
$$

If we assume $\operatorname{Im} F(\hat{\omega})=0$ for any $\omega \in \mathbb{S}^{1}$ in addition, then $P(\tau, \sigma, \omega)=$ $P_{0}(\sigma, \omega)$. Furthermore we see from (2.3) that $P_{0} \in L^{2}\left(\mathbb{R} \times \mathbb{S}^{1}\right)$. Hence we conclude that the solution $u$ is asymptotically free. For this case, we have $F(\hat{\omega}) \equiv 0$ and the cubic null condition (for the complex case) is satisfied. Typical examples are $F=\left(\partial_{a} u\right)\left(\left|\partial_{t} u\right|^{2}-\left|\partial_{1} u\right|^{2}-\left|\partial_{2} u\right|^{2}\right)$ or $\left(\overline{\partial_{a} u}\right)\left(\left(\partial_{t} u\right)^{2}-\right.$ $\left.\left(\partial_{1} u\right)^{2}-\left(\partial_{2} u\right)^{2}\right)$, as well as $F=\left(\partial_{a} u\right)\left(\left(\partial_{b} u\right)\left(\overline{\partial_{c} u}\right)-\left(\partial_{c} u\right)\left(\overline{\partial_{b} u}\right)\right)$ for $a, b, c=$ $0,1,2$.

The situation is different if $\operatorname{Im} F(\hat{\omega}) \not \equiv 0$. For example, take $F(\partial u)=$ $i\left|\partial_{t} u\right|^{2}\left(\partial_{t} u\right)$ so that $F(\hat{\omega}) \equiv-i$. Then it is easy to show $\|u(t)\|_{E}=\|u(0)\|_{E}$. By (2.12) we get

$$
P(\tau, \sigma, \omega)=P_{0}(\sigma, \omega) \exp \left(\frac{i}{2}\left|P_{0}(\sigma, \omega)\right|^{2} \tau\right),
$$

and we can easily see that $P(\tau)$ does not converge to any function in $L^{2}\left(\mathbb{R} \times \mathbb{S}^{1}\right)$ as $\tau \rightarrow \infty$ unless $P_{0} \equiv 0$. Because of the conservation of the energy, we can show that if $P_{0} \equiv 0$ then $(f, g) \equiv(0,0)$. Hence we see that the global solution $u$ for small $\varepsilon$ is not asymptotically free unless $(f, g) \equiv(0,0)$, though the energy is preserved. Such a phenomenon never occurs in the real-valued case. 


\section{Preliminaries}

We introduce

$$
\begin{aligned}
& S:=t \partial_{t}+\sum_{j=1}^{2} x_{j} \partial_{j} \\
& L=\left(L_{1}, L_{2}\right):=\left(t \partial_{1}+x_{1} \partial_{t}, t \partial_{2}+x_{2} \partial_{t}\right), \Omega:=x_{1} \partial_{2}-x_{2} \partial_{1},
\end{aligned}
$$

and we set

$$
\Gamma=\left(\Gamma_{0}, \Gamma_{1}, \ldots, \Gamma_{6}\right)=\left(S, L_{1}, L_{2}, \Omega, \partial_{0}, \partial_{1}, \partial_{2}\right) .
$$

With a multi-index $\alpha=\left(\alpha_{0}, \alpha_{1}, \ldots, \alpha_{6}\right) \in \mathbb{N}_{0}^{7}$, we write $\Gamma^{\alpha}=\Gamma_{0}^{\alpha_{0}} \Gamma_{1}^{\alpha_{1}} \cdots \Gamma_{6}^{\alpha_{6}}$, where $\mathbb{N}_{0}$ denotes the set of nonnegative integers. For a smooth function $\psi=\psi(t, x)$ and a nonnegative integer $s$, we define

$$
|\psi(t, x)|_{s}=\sum_{|\alpha| \leq s}\left|\Gamma^{\alpha} \psi(t, x)\right|, \quad\|\psi(t)\|_{s}=\sum_{|\alpha| \leq s}\left\|\Gamma^{\alpha} \psi(t, \cdot)\right\|_{L^{2}\left(\mathbb{R}^{2}\right)} .
$$

It is easy to see that $[\square, \Omega]=\left[\square, L_{j}\right]=\left[\square, \partial_{a}\right]=0$ for $j=1,2$ and $a=0,1,2$, where $[A, B]=A B-B A$ for the operators $A$ and $B$. We also have $[\square, S]=2 \square$. Therefore for any $\alpha=\left(\alpha_{0}, \alpha_{1}, \ldots, \alpha_{6}\right) \in \mathbb{N}_{0}^{7}$ and a smooth function $\psi$, we have

$$
\square \Gamma^{\alpha} \psi=\left(\Gamma_{0}+2\right)^{\alpha_{0}} \Gamma_{1}^{\alpha_{1}} \cdots \Gamma_{6}^{\alpha_{6}} \square \psi=: \widetilde{\Gamma}^{\alpha} \square \psi .
$$

We can check that we have $\left[\Gamma_{a}, \Gamma_{b}\right]=\sum_{0 \leq c \leq 7} A_{c}^{a b} \Gamma_{c}$ and $\left[\Gamma_{a}, \partial_{b}\right]=\sum_{0 \leq c \leq 2} B_{c}^{a b} \partial_{c}$ with appropriate constants $A_{c}^{a b}$ and $B_{c}^{a \bar{b}}$. Hence for any $\alpha, \beta \in \mathbb{N}_{0}^{7}$, and any nonnegative integer $s$, there exist positive constants $C_{\alpha, \beta}$ and $C_{s}$ such that

$$
\begin{aligned}
& \left|\Gamma^{\alpha} \Gamma^{\beta} \psi(t, x)\right| \leq C_{\alpha, \beta}|\psi(t, x)|_{|\alpha|+|\beta|}, \\
& C_{s}^{-1}|\partial \psi(t, x)|_{s} \leq \sum_{0 \leq a \leq 2} \sum_{|\gamma| \leq s}\left|\partial_{a} \Gamma^{\gamma} \psi(t, x)\right| \leq C_{s}|\partial \psi(t, x)|_{s}
\end{aligned}
$$

for any smooth function $\psi$.

Using these vector fields, we obtain a good decay estimate for the solution to the inhomogeneous wave equation. Let $0<T \leq \infty$.

Lemma 3.1 (Hörmander's $L^{1}-L^{\infty}$ estimate). Let $v$ be a smooth solution to

$$
\square v(t, x)=\Psi(t, x), \quad(t, x) \in(0, T) \times \mathbb{R}^{2}
$$

with initial data $v=\partial_{t} v=0$ at $t=0$. Then there exists a universal positive constant $C$ such that

$$
\langle t+|x|\rangle^{1 / 2}|v(t, x)| \leq C \int_{0}^{t}\left(\int_{\mathbb{R}^{2}} \frac{|\Psi(\tau, y)|_{1}}{\langle\tau+|y|\rangle^{1 / 2}} d y\right) d \tau, \quad 0 \leq t<T .
$$

See Hörmander [6] for the proof (similar estimates for arbitrary space dimensions are also available there).

The vector fields in $\Gamma$ also play an important role in the reduction of the analysis of semilinear wave equations to that of the corresponding ordinary differential equations (or ODEs in short). We use the polar coordinates $(r, \theta)$ to write $x=\left(x_{1}, x_{2}\right)=(r \cos \theta, r \sin \theta)$ with $r=|x|$ and $\theta \in \mathbb{R}$. We put 
$\omega=\left(\omega_{1}, \omega_{2}\right)=x /|x|=(\cos \theta, \sin \theta)$. Then we have $\partial_{r}=\sum_{j=1}^{2} \omega_{j} \partial_{j}$, and $\partial_{\theta}=x_{1} \partial_{2}-x_{2} \partial_{1}=\Omega$. We also have

$$
\partial_{1}=\omega_{1} \partial_{r}-\frac{\omega_{2}}{r} \partial_{\theta}, \quad \partial_{2}=\omega_{2} \partial_{r}+\frac{\omega_{1}}{r} \partial_{\theta}
$$

We put

$$
\partial_{ \pm}=\partial_{t} \pm \partial_{r}
$$

Then, for a smooth function $\psi$, we get

$$
r^{1 / 2} \square \psi=\partial_{+} \partial_{-}\left(r^{1 / 2} \psi\right)-r^{-3 / 2}\left(\partial_{\theta}^{2} \psi+\frac{1}{4} \psi\right) .
$$

Since

$$
\frac{1}{r} \partial_{\theta}=\frac{1}{r}\left(x_{1} \partial_{2}-x_{2} \partial_{1}\right)=\frac{1}{t}\left(\omega_{1} L_{2}-\omega_{2} L_{1}\right),
$$

it follows from (3.4) that

$$
\sum_{j=1}^{2}\left|\partial_{j} \psi(t, x)-\omega_{j} \partial_{r} \psi(t, x)\right| \leq\left|\frac{1}{r} \partial_{\theta} \psi(t, x)\right| \leq C\langle t+r\rangle^{-1}|\psi(t, x)|_{1}
$$

for any $\psi \in C^{1}\left([0, T) \times \mathbb{R}^{2}\right)$.

We put $\omega_{0}=-1$ and $\hat{\omega}=\left(\omega_{0}, \omega_{1}, \omega_{2}\right)$. For simplicity of exposition, we introduce

$$
D_{ \pm}= \pm \frac{1}{2} \partial_{ \pm}=\frac{1}{2}\left(\partial_{r} \pm \partial_{t}\right)
$$

Lemma 3.2. There exists a positive constant $C$ such that

$$
\left|\partial \psi(t, x)-\hat{\omega} D_{-} \psi(t, x)\right| \leq C\langle t+r\rangle^{-1}|\psi(t, x)|_{1}
$$

for $\psi \in C^{1}\left([0, T) \times \mathbb{R}^{2}\right)$ and $(t, x) \in[0, T) \times\left(\mathbb{R}^{2} \backslash\{0\}\right)$.

Proof. Because $S=t \partial_{t}+r \partial_{r}$, if we put $L_{r}=t \partial_{r}+r \partial_{t}=\sum_{j=1}^{2} \omega_{j} L_{j}$, then we get

$$
D_{+}=\frac{1}{2}\left(\partial_{r}+\partial_{t}\right)=\frac{1}{2(t+r)}\left(S+L_{r}\right) \text {. }
$$

Since we have $\partial_{t}=-D_{-}+D_{+}$and $\partial_{r}=D_{-}+D_{+}$, we find that $\mid \partial_{t} \psi(t, x)-$ $\omega_{0} D_{-} \psi(t, x) \mid$ and $\left|\partial_{r} \psi(t, x)-D_{-} \psi(t, x)\right|$ are bounded by $C\langle t+r\rangle^{-1}|\psi(t, x)|_{1}$. Hence we obtain (3.7) with the help of (3.6).

We define

$$
\Lambda_{T}:=\left\{(t, x) \in[0, T) \times \mathbb{R}^{2} ; r \geq t / 2 \geq 1\right\} .
$$

Observe that we have $r^{-1} \leq 4(1+t+r)^{-1}$ for $(t, x) \in \Lambda_{T}$. We compute $D_{-}\left(r^{1 / 2} \psi\right)=r^{1 / 2} D_{-} \psi+r^{-1 / 2} \psi / 4$. Thus from (3.7) we obtain the following.

Corollary 3.3. There is a positive constant $C$ such that

$$
\left|r^{1 / 2} \partial \psi(t, x)-\hat{\omega} D_{-}\left(r^{1 / 2} \psi(t, x)\right)\right| \leq C\langle t+r\rangle^{-1 / 2}|\psi(t, x)|_{1}
$$

for $(t, x) \in \Lambda_{T}$ and $\psi \in C^{1}\left([0, T) \times \mathbb{R}^{2}\right)$.

The following lemma is due to Lindblad [16]. 
Lemma 3.4. For any nonnegative integer $s$, there exists a positive constant $C_{s}$ such that

$$
|\partial \psi(t, x)|_{s} \leq C_{s}\langle t-r\rangle^{-1}|\psi(t, x)|_{s+1}, \quad(t, x) \in[0, T) \times \mathbb{R}^{2}
$$

for any $\psi \in C^{s+1}\left([0, T) \times \mathbb{R}^{2}\right)$.

Proof. In view of (3.2) and (3.3), it suffices to prove (3.8) for $s=0$. As in the proof of Lemma 3.2, we put $L_{r}=t \partial_{r}+r \partial_{t}$. Then we have

$$
(t-r) \partial_{t} \psi=\frac{1}{t+r}\left(t S-r L_{r}\right) \psi, \quad(t-r) \partial_{r} \psi=\frac{1}{t+r}\left(t L_{r}-r S\right) \psi
$$

Hence we get $\langle t-r\rangle\left(\left|\partial_{t} \psi\right|+\left|\partial_{r} \psi\right|\right) \leq C|\psi|_{1}$, which leads to (3.8) for $s=0$ with the help of (3.6).

\section{Reduction to Simplified EQUATIONS}

Let $0<T \leq \infty$, and let $u$ be the solution to (1.1) on $[0, T) \times \mathbb{R}^{2}$. We suppose that

$$
\operatorname{supp} f \cup \operatorname{supp} g \subset B_{R}
$$

for some $R>0$, where $B_{M}=\left\{x \in \mathbb{R}^{2} ;|x| \leq M\right\}$ for $M>0$. Then, from the finite propagation property, we have

$$
\operatorname{supp} u(t, \cdot) \subset B_{t+R}, \quad 0 \leq t<T .
$$

In what follows, we put $r=|x|, \omega=\left(\omega_{1}, \omega_{2}\right)=|x|^{-1} x$, and $\omega_{0}=-1$. We write $\hat{\omega}=\left(\omega_{0}, \omega\right)=(-1, \omega)$. We define

$$
U(t, x):=D_{-}\left(r^{1 / 2} u(t, x)\right), \quad(t, x) \in[0, T) \times\left(\mathbb{R}^{2} \backslash\{0\}\right) .
$$

From (1.1) and (3.5), we get

$$
\partial_{+} U(t, x)=-\frac{1}{2 t} F(\hat{\omega})|U(t, x)|^{2} U(t, x)+H(t, x),
$$

where $H=H(t, x)$ is given by

$$
H=-\frac{1}{2}\left(r^{1 / 2} F(\partial u)-t^{-1} F(\hat{\omega})|U|^{2} U\right)-\frac{1}{2 r^{3 / 2}}\left(\partial_{\theta}^{2} u+\frac{1}{4} u\right) .
$$

(4.3) plays an important role in our analysis.

We also need the following to estimate the generalized derivative $\Gamma^{\alpha} u$ for a multi-index $\alpha=\left(\alpha_{0}, \alpha_{1}, \ldots, \alpha_{6}\right) \in \mathbb{N}_{0}^{7}$ : (3.1) and (1.1) yield $\square\left(\Gamma^{\alpha} u\right)=$ $\widetilde{\Gamma}^{\alpha}(F(\partial u))$, which leads to

$$
\partial_{+} U^{(\alpha)}=-\frac{1}{2 t} F(\hat{\omega})\left(2|U|^{2} U^{(\alpha)}+U^{2} \overline{U^{(\alpha)}}\right)+H_{\alpha},
$$

where we have put

$$
U^{(\alpha)}(t, x):=D_{-}\left(r^{1 / 2} \Gamma^{\alpha} u(t, x)\right),
$$


and $H_{\alpha}=H_{\alpha}(t, x)$ is given by

$$
\begin{aligned}
H_{\alpha}= & -\frac{1}{2}\left(r^{1 / 2} \widetilde{\Gamma}^{\alpha} F(\partial u)-t^{-1} F(\hat{\omega})\left(2|U|^{2} U^{(\alpha)}+U^{2} \overline{U^{(\alpha)}}\right)\right) \\
& -\frac{1}{2 r^{3 / 2}}\left(\partial_{\theta}^{2} \Gamma^{\alpha} u+\frac{1}{4} \Gamma^{\alpha} u\right) .
\end{aligned}
$$

Note that $U=U^{(\alpha)}$ if $|\alpha|=0$.

We put

$$
\Lambda_{T, R}:=\left\{(t, x) \in[0, T) \times \mathbb{R}^{2} ; 1 \leq t / 2 \leq r \leq t+R\right\} .
$$

Note that we have

$$
(1+t+r)^{-1} \leq r^{-1} \leq 2 t^{-1} \leq 3(1+t)^{-1} \leq 3(R+2)(1+t+r)^{-1}
$$

for $(t, x) \in \Lambda_{T, R}$. In other words, the weights $\langle t+r\rangle^{-1},(1+t)^{-1}, r^{-1}$, and $t^{-1}$ are equivalent to each other in $\Lambda_{T, R}$. For nonnegative integer $s$, we define

$$
|u(t, x)|_{\sharp, s}:=|\partial u(t, x)|_{s}+\langle t+r\rangle^{-1}|u(t, x)|_{s+1} .
$$

For $|\alpha| \leq s$, Corollary 3.3 and (3.3) immediately imply that

$$
\left|U^{(\alpha)}(t, x)\right| \leq C_{s} r^{1 / 2}|u(t, x)|_{\sharp, s}
$$

for $(t, x) \in \Lambda_{T, R}$ with some positive constant $C_{s}$. Our final goal in this section is to prove the following.

Proposition 4.1. Suppose that (2.1) is fulfilled. Let $s$ be a positive integer, and suppose that $1 \leq|\alpha| \leq s$. Then there are positive constants $C$ and $C_{s}$ such that

$$
\begin{aligned}
|H(t, x)| & \leq C\left(t^{-1 / 2}|u|_{\sharp, 0}^{2}|u|_{1}+t^{-3 / 2}|u|_{2}\right), \\
\left|H_{\alpha}(t, x)\right| & \leq C_{s}\left(t^{-1 / 2}|u|_{\sharp, s}^{2}|u|_{s+1}+r^{1 / 2}|\partial u|_{s-1}^{3}+t^{-3 / 2}|u|_{s+2}\right)
\end{aligned}
$$

for $(t, x) \in \Lambda_{T, R}$.

Before we proceed to the proof of Proposition 4.1, we show one lemma. For $a, b, c=0,1,2$, we define

$$
Q_{a b c}:=r^{1 / 2}\left(\partial_{a} u\right)\left(\partial_{b} u\right)\left(\overline{\partial_{c} u}\right)-r^{-1} \omega_{a} \omega_{b} \omega_{c}|U|^{2} U
$$

For a multi-index $\alpha \in \mathbb{N}_{0}^{7}$, we also define

$$
\widetilde{Q}_{a b c}^{(\alpha)}:=r^{1 / 2} \Gamma^{\alpha}\left(\left(\partial_{a} u\right)\left(\partial_{b} u\right)\left(\overline{\partial_{c} u}\right)\right)-r^{-1} \omega_{a} \omega_{b} \omega_{c}\left(2|U|^{2} U^{(\alpha)}+U^{2} \overline{U^{(\alpha)}}\right) .
$$

Lemma 4.2. Let $|\alpha| \leq s$. Then there are positive constants $C$ and $C_{s}$ such that

$$
\begin{aligned}
& \left|Q_{a b c}\right| \leq C t^{-1 / 2}|u|_{\sharp, 0}^{2}|u|_{1}, \\
& \left|\widetilde{Q}_{a b c}^{(\alpha)}\right| \leq C_{s}\left(t^{-1 / 2}|u|_{\sharp, s}^{2}|u|_{s+1}+r^{1 / 2}|\partial u|_{s-1}^{3}\right)
\end{aligned}
$$

for $(t, x) \in \Lambda_{T, R}$. 
Proof. Suppose that $(t, x) \in \Lambda_{T, R}$. By (4.7) and Corollary 3.3, we get

$$
\begin{aligned}
\left|Q_{a b c}\right| & =r^{-1}\left(\left(r^{1 / 2} \partial_{a} u\right)\left(r^{1 / 2} \partial_{b} u\right)\left(\overline{r^{1 / 2} \partial_{c} u}\right)-\omega_{a} \omega_{b} \omega_{c}|U|^{2} U\right) \\
& \leq C r^{-1}\left(\left|r^{1 / 2} \partial u\right|+|U|\right)^{2}\left|r^{1 / 2} \partial u-\hat{\omega} U\right| \\
& \leq C t^{-1 / 2}|u|_{\sharp, 0}^{2}|u|_{1} .
\end{aligned}
$$

Let $|\alpha| \leq s$. In view of $(3.2)$ and (3.3) we obtain

$$
\left|\Gamma^{\alpha}\left(\left(\partial_{a} u\right)\left(\partial_{b} u\right)\left(\overline{\partial_{c} u}\right)\right)-R_{a b c}^{(\alpha)}\right| \leq C_{s}|\partial u|_{[s / 2]}^{2}|\partial u|_{s-1} \leq C_{s}|\partial u|_{s-1}^{3}
$$

with some positive integer $C_{s}$, where

$$
R_{a b c}^{(\alpha)}=\left(\partial_{a} \Gamma^{\alpha} u\right)\left(\partial_{b} u\right)\left(\overline{\partial_{c} u}\right)+\left(\partial_{a} u\right)\left(\partial_{b} \Gamma^{\alpha} u\right)\left(\overline{\partial_{c} u}\right)+\left(\partial_{a} u\right)\left(\partial_{b} u\right)\left(\overline{\partial_{c} \Gamma^{\alpha} u}\right) .
$$

Similarly to (4.12), we get

$$
\left|r^{1 / 2} R_{a b c}^{(\alpha)}-r^{-1} \omega_{a} \omega_{b} \omega_{c}\left(2|U|^{2} U^{(\alpha)}+U^{2} \overline{U^{(\alpha)}}\right)\right| \leq C_{s} t^{-1 / 2}|u|_{\sharp, s}^{2}|u|_{s+1} .
$$

The desired estimate (4.11) follows from (4.13) and (4.14).

Proof of Proposition 4.1. Suppose that $(t, x) \in \Lambda_{T, R}$. Recalling (2.1), we get $r^{1 / 2} F(\partial u)-r^{-1} F(\hat{\omega})|U|^{2} U=\sum_{a, b, c} p_{a b c} Q_{a b c}$, and Lemma 4.2 yields

$$
\left.\left.\left|r^{1 / 2} F(\partial u)-r^{-1} F(\hat{\omega})\right| U\right|^{2} U\left|\leq C t^{-1 / 2}\right| u\right|_{\sharp, 0} ^{2}|u|_{1} .
$$

Using (4.7) and Lemma 3.4 we get

$$
\begin{aligned}
\left.\left|\left(r^{-1}-t^{-1}\right) F(\hat{\omega})\right| U\right|^{2} U \mid & \leq C t^{-1} r^{-1}\langle t-r\rangle r^{3 / 2}|u|_{\sharp, 0}^{3} \\
& \leq C t^{-1 / 2}|u|_{\sharp, 0}^{2}\left(\langle t-r\rangle|\partial u|+|u|_{1}\right) \\
& \leq C t^{-1 / 2}|u|_{\sharp, 0}^{2}|u|_{1} .
\end{aligned}
$$

It is clear that we have

$$
r^{-3 / 2}\left|\partial_{\theta}^{2} u+u / 4\right| \leq C t^{-3 / 2}|u|_{2} .
$$

Now (4.8) follows from (4.15), (4.16), and (4.17).

Using (4.11) instead of (4.10), we can show (4.9) in a similar manner.

\section{A KEY LEMMA FOR ORDINARY DIFFERENTIAL EQUATIONS}

Let $t_{0} \geq 1$. Motivated by (4.3), we consider the initial value problem for the following kind of ODE:

$$
\begin{aligned}
& z^{\prime}(t)=-\frac{K}{2 t}|z(t)|^{2} z(t)+J(t), \quad t_{0}<t<\infty \\
& z\left(t_{0}\right)=z_{0}
\end{aligned}
$$

where $K, z_{0} \in \mathbb{C}$, and $J \in C\left(\left[t_{0}, \infty\right) ; \mathbb{C}\right)$. The following lemma is a refinement of the ones obtained in Hayashi-Naumkin-Sunagawa [5] and Sunagawa [20]. 
Lemma 5.1. Let $\varepsilon>0, \sigma \in \mathbb{R}, \rho>1,0<\mu<\rho-1$, and $\kappa \geq 0$. Suppose that $\operatorname{Re} K \geq 0$. We assume that there are positive constants $K_{0}$ and $E_{0}$ such that

$$
\begin{aligned}
|K| & \leq K_{0} \\
\left|z_{0}\right| & \leq E_{0} \varepsilon\langle\sigma\rangle^{-\kappa-\rho+1}, \\
|J(t)| & \leq E_{0} \varepsilon\langle\sigma\rangle^{-\kappa} t^{-\rho}, \quad t \geq t_{0} .
\end{aligned}
$$

We also assume that there is a positive constant $c_{0}$ such that

$$
c_{0}^{-1}\langle\sigma\rangle<t_{0}<c_{0}\langle\sigma\rangle \text {. }
$$

Then (5.1) -(5.2) admits a unique global solution $z \in C^{1}\left(\left[t_{0}, \infty\right) ; \mathbb{C}\right)$, and there is a positive constant $C_{0}=C_{0}\left(E_{0}, c_{0}, \rho\right)$ such that we have

$$
|z(t)| \leq C_{0} \varepsilon\langle\sigma\rangle^{-\kappa-\rho+1}, \quad t_{0} \leq t<\infty .
$$

Moreover there is a positive constant $\varepsilon_{1}=\varepsilon_{1}\left(K_{0}, E_{0}, c_{0}, \rho, \mu\right)$ such that if $0<$ $\varepsilon \leq \varepsilon_{1}$, then we can find $p_{0} \in \mathbb{C}$ satisfying

$$
\begin{aligned}
|z(t)-p(\log t)| & \leq C_{1} \varepsilon\langle\sigma\rangle^{-\kappa-\mu} t^{-\rho+\mu+1}, \quad t \geq t_{0}, \\
\left|p_{0}\right| & \leq C_{1} \varepsilon\langle\sigma\rangle^{-\kappa-\rho+1}
\end{aligned}
$$

where $p(t)$ is a solution to

$$
\left\{\begin{array}{l}
p^{\prime}(\tau)=-\frac{K}{2}|p(\tau)|^{2} p(\tau), \quad \tau>0, \\
p(0)=p_{0},
\end{array}\right.
$$

and $C_{1}=C_{1}\left(K_{0}, E_{0}, c_{0}, \rho, \mu\right)$ is a positive constant.

Proof. We put $\nu=\kappa+\rho-1(>0)$. Let $z$ be the solution to (5.1)-(5.2) for $t_{0}<t<T_{0}$ with some $T_{0}\left(>t_{0}\right)$. Since $\operatorname{Re} K \geq 0$, it follows from (5.1) that

$$
\frac{d}{d t}\left(|z(t)|^{2}\right)=-\frac{\operatorname{Re} K}{t}|z(t)|^{4}+2 \operatorname{Re}(J(t) \overline{z(t)}) \leq 2|J(t)||z(t)|,
$$

which yields

$$
\begin{aligned}
|z(t)| & \leq\left|z_{0}\right|+\int_{t_{0}}^{t}|J(\tau)| d \tau \leq E_{0} \varepsilon\left(\langle\sigma\rangle^{-\nu}+\langle\sigma\rangle^{-\kappa} \int_{t_{0}}^{\infty} \tau^{-\rho} d \tau\right) \\
& =E_{0} \varepsilon\left(\langle\sigma\rangle^{-\nu}+\langle\sigma\rangle^{-\kappa} \frac{t_{0}^{-\rho+1}}{\rho-1}\right) \leq E_{0}\left(1+\frac{c_{0}^{\rho-1}}{\rho-1}\right) \varepsilon\langle\sigma\rangle^{-\nu}
\end{aligned}
$$

for $t_{0} \leq t<T_{0}$. With this a priori bound and the local existence theorem for ODEs, we can easily show the global existence of the unique solution $z$ to (5.1) -(5.2), and we obtain (5.4).

Now we turn our attention to the asymptotic behavior of $z$. In what follows, $C$ stands for various positive constants that can be determined only by $K_{0}$, $E_{0}, c_{0}, \rho$, and $\mu$. The actual value of $C$ may change line by line. 
Let $\xi$ and $\eta$ be the solution to the system of ODEs

$$
\begin{array}{llrl}
\xi^{\prime}(t) & =-i \frac{\operatorname{Im} K}{2 t \eta(t)}|\xi(t)|^{2} \xi(t)+J(t) \sqrt{\eta(t)}, & & t>t_{0}, \\
\eta^{\prime}(t)=\frac{\operatorname{Re} K}{t}|\xi(t)|^{2}, & & t>t_{0}, \\
\xi\left(t_{0}\right)=z_{0}, \quad \eta\left(t_{0}\right)=1 . &
\end{array}
$$

We can easily check that $z(t)=\xi(t) / \sqrt{\eta(t)}$ as long as $(\xi, \eta)$ exists.

By the local existence theorem for ODEs, there is a local solution $(\xi, \eta)$ on $\left[t_{0}, T_{1}\right)$ with some $T_{1}\left(>t_{0}\right)$. For $t_{0}<\tau_{0}<T_{1}$, we put

$$
M_{\tau_{0}}:=\sup _{t_{0} \leq t \leq \tau_{0}}|\xi(t)| \text {. }
$$

Then we get $0 \leq \eta^{\prime}(t) \leq K_{0} M_{\tau_{0}}^{2} t^{-1}$ for $t_{0} \leq t \leq \tau_{0}$, which leads to

$$
1 \leq \eta(t) \leq 1+K_{0} M_{\tau_{0}}^{2} \log \left(t / t_{0}\right), \quad t_{0} \leq t \leq \tau_{0}
$$

By (5.8) we obtain

$$
\frac{d}{d t}|\xi(t)|^{2}=2 \operatorname{Re}(\overline{\xi(t)} J(t) \sqrt{\eta(t)}) \leq 2|\xi(t)||J(t)| \sqrt{\eta(t)}
$$

which, in combination with (5.11), yields

$$
\begin{aligned}
|\xi(t)| & \leq\left|z_{0}\right|+\int_{t_{0}}^{t}|J(\tau)| \sqrt{\eta(\tau)} d \tau \\
& \leq C \varepsilon\left(\langle\sigma\rangle^{-\nu}+\langle\sigma\rangle^{-\kappa} \int_{t_{0}}^{t} \tau^{-\rho}\left(1+M_{\tau_{0}} \sqrt{\log \frac{\tau}{t_{0}}}\right) d \tau\right)
\end{aligned}
$$

for $t_{0} \leq t \leq \tau_{0}$. Since we have $\log t \leq C_{\mu} t^{2 \mu}$ for $t \geq 1$ with a positive constant $C_{\mu}$, and since $-\rho+\mu+1<0$, we get

$$
\int_{t_{0}}^{t} \tau^{-\rho} \sqrt{\log \frac{\tau}{t_{0}}} d \tau \leq C t_{0}^{-\mu} \int_{t_{0}}^{\infty} \tau^{-\rho+\mu} d \tau \leq C t_{0}^{-\rho+1} \leq C\langle\sigma\rangle^{-\rho+1}, \quad t \geq t_{0} .
$$

Hence we obtain from (5.12) that

$$
M_{\tau_{0}} \leq C \varepsilon\langle\sigma\rangle^{-\nu}+C \varepsilon\langle\sigma\rangle^{-\nu} M_{\tau_{0}} \leq C \varepsilon\langle\sigma\rangle^{-\nu}+\frac{1}{2} M_{\tau_{0}}
$$

for $0<\varepsilon \leq \varepsilon_{2}:=1 /(2 C)$ (with the constant $C$ from (15.13) $)$, which yields

$$
\left(\sup _{t_{0} \leq t \leq \tau_{0}}|\xi(t)|=\right) M_{\tau_{0}} \leq C \varepsilon\langle\sigma\rangle^{-\nu}
$$

provided that $0<\varepsilon \leq \varepsilon_{2}$. With the a priori bound (5.14) as well as (5.11), we see that the solution $(\xi, \eta)$ exists globally in time, and we also have

$$
|\xi(t)| \leq C \varepsilon\langle\sigma\rangle^{-\nu}, 1 \leq \eta(t) \leq 1+C \varepsilon^{2} \log \frac{t}{t_{0}} \leq 1+C \varepsilon^{2}\left(\frac{t}{t_{0}}\right)^{2 \mu}
$$

for $t \geq t_{0}$, provided that $0<\varepsilon \leq \varepsilon_{2}$. 
We assume $0<\varepsilon \leq \varepsilon_{2}$ from now on. We put

$$
\Theta(t)=\frac{\operatorname{Im} K}{2} \int_{t_{0}}^{t} \frac{|\xi(\tau)|^{2}}{\tau \eta(\tau)} d \tau, \quad t \geq t_{0}
$$

Then (5.8) implies

$$
\xi(t)=e^{-i \Theta(t)}\left(z_{0}+\int_{t_{0}}^{t} e^{i \Theta(\tau)} J(\tau) \sqrt{\eta(\tau)} d \tau\right), \quad t \geq t_{0} .
$$

We define

$$
z_{+}:=z_{0}+\int_{t_{0}}^{\infty} e^{i \Theta(\tau)} J(\tau) \sqrt{\eta(\tau)} d \tau, \quad \xi_{+}(t):=e^{-i \Theta(t)} z_{+} .
$$

Then we obtain

$$
\begin{aligned}
\left|\xi_{+}(t)-\xi(t)\right| & \leq \int_{t}^{\infty}|J(\tau)| \sqrt{\eta(\tau)} d \tau \\
& \leq C \varepsilon\langle\sigma\rangle^{-\kappa} \int_{t}^{\infty} \tau^{-\rho}\left(1+\varepsilon_{2}\left(\frac{\tau}{t_{0}}\right)^{\mu}\right) d \tau \\
& \leq C \varepsilon\left(\langle\sigma\rangle^{-\kappa} t^{-\rho+1}+\langle\sigma\rangle^{-\kappa-\mu} t^{-\rho+\mu+1}\right) \\
& \leq C \varepsilon\langle\sigma\rangle^{-\kappa-\mu} t^{-\rho+\mu+1}, \quad t \geq t_{0} .
\end{aligned}
$$

Especially we have

$$
\left|z_{+}-z_{0}\right| \leq C \varepsilon\langle\sigma\rangle^{-\kappa-\mu} t_{0}^{-\rho+\mu+1} \leq C \varepsilon\langle\sigma\rangle^{-\nu},
$$

which shows

$$
\left|z_{+}\right| \leq C \varepsilon\langle\sigma\rangle^{-\nu}
$$

Observing that $\left|z_{+}\right|=\left|\xi_{+}(t)\right|$, we obtain from (15.16) and (5.17) that

$$
\left.|| \xi(t)\right|^{2}-\left|z_{+}\right|^{2}\left|\leq\left(|\xi(t)|+\left|z_{+}\right|\right)\right| \xi(t)-\xi_{+}(t) \mid \leq C \varepsilon^{2}\langle\sigma\rangle^{-\nu-\kappa-\mu} t^{-\rho+\mu+1}
$$

for $t \geq t_{0}$. We define

$$
\eta_{\infty}(t):=1+(\operatorname{Re} K)\left(\left|z_{+}\right|^{2} \log \frac{t}{t_{0}}+\int_{t_{0}}^{\infty} \frac{|\xi(\tau)|^{2}-\left|z_{+}\right|^{2}}{\tau} d \tau\right), \quad t \geq 1 .
$$

Since (5.9) implies that

$$
\eta(t)=1+(\operatorname{Re} K)\left(\left|z_{+}\right|^{2} \log \frac{t}{t_{0}}+\int_{t_{0}}^{t} \frac{|\xi(\tau)|^{2}-\left|z_{+}\right|^{2}}{\tau} d \tau\right), \quad t \geq t_{0}
$$

we obtain from (5.18) that

$$
\left|\eta(t)-\eta_{\infty}(t)\right| \leq K_{0} \int_{t}^{\infty} \frac{\left.|| \xi(\tau)\right|^{2}-\left|z_{+}\right|^{2} \mid}{\tau} d \tau \leq C \varepsilon^{2}\langle\sigma\rangle^{-\nu-\kappa-\mu} t^{-\rho+\mu+1}
$$

for $t \geq t_{0}$. Especially we have $\eta_{\infty}\left(t_{0}\right) \geq 1-C \varepsilon^{2}$, which leads to

$$
\eta_{\infty}(t) \geq 1-C \varepsilon^{2}-K_{0}\left|z_{+}\right|^{2} \log t_{0} \geq 1-C \varepsilon^{2}-C \varepsilon^{2}\langle\sigma\rangle^{-2 \nu} \log t_{0}, \quad t \geq 1
$$

with the help of (5.17). By (5.3), there is a positive constant $c_{1}=c_{1}\left(c_{0}, \rho\right)$ such that we have

$$
\langle\sigma\rangle^{-2 \nu} \log t_{0} \leq\langle\sigma\rangle^{-2(\rho-1)} \log \left(c_{0}\langle\sigma\rangle\right) \leq c_{1}, \quad \sigma \in \mathbb{R} .
$$


If we put

$$
\varepsilon_{1}:=\min \left\{\sqrt{\frac{1}{4 C}}, \sqrt{\frac{1}{4 c_{1} C}}, \varepsilon_{2}\right\}
$$

with the constant $C$ coming from (5.20), then we get

$$
\eta_{\infty}(t) \geq \frac{1}{2}, \quad t \geq 1
$$

for $0<\varepsilon \leq \varepsilon_{1}$.

From now on, we assume that $0<\varepsilon \leq \varepsilon_{1}$. We set

$$
\begin{aligned}
\Theta_{\infty}(t) & :=\frac{\operatorname{Im} K}{2} \int_{t_{0}}^{t} \frac{\left|z_{+}\right|^{2}}{\tau \eta_{\infty}(\tau)} d \tau, \quad t \geq 1 \\
\Theta_{0} & :=\frac{\operatorname{Im} K}{2} \int_{t_{0}}^{\infty}\left(\frac{|\xi(\tau)|^{2}}{\tau \eta(\tau)}-\frac{\left|z_{+}\right|^{2}}{\tau \eta_{\infty}(\tau)}\right) d \tau .
\end{aligned}
$$

From (5.15), (5.17), (5.18), (5.19), and (5.21), we obtain

$$
\begin{aligned}
\left|\frac{|\xi(\tau)|^{2}}{\eta(\tau)}-\frac{\left|z_{+}\right|^{2}}{\eta_{\infty}(\tau)}\right| & \leq\left|\frac{|\xi(\tau)|^{2}-\left|z_{+}\right|^{2}}{\eta(\tau)}\right|+\left|z_{+}\right|^{2}\left|\frac{1}{\eta(\tau)}-\frac{1}{\eta_{\infty}(\tau)}\right| \\
& \leq C \varepsilon^{2}\langle\sigma\rangle^{-\nu-\kappa-\mu} \tau^{-\rho+\mu+1}
\end{aligned}
$$

for $\tau \geq t_{0}$, which yields

$$
\begin{aligned}
\left|\left(\Theta_{\infty}(t)+\Theta_{0}\right)-\Theta(t)\right| & \leq \frac{K_{0}}{2} \int_{t}^{\infty}\left|\frac{|\xi(\tau)|^{2}}{\tau \eta(\tau)}-\frac{\left|z_{+}\right|^{2}}{\tau \eta_{\infty}(\tau)}\right| d \tau \\
& \leq C \varepsilon^{2}\langle\sigma\rangle^{-\nu-\kappa-\mu} t^{-\rho+\mu+1}, \quad t \geq t_{0} .
\end{aligned}
$$

We define

$$
\xi_{\infty}(t):=e^{-i \Theta_{\infty}(t)}\left(e^{-i \Theta_{0}} z_{+}\right), \quad t \geq 1 .
$$

Then (5.22) leads to

$$
\begin{aligned}
\left|\xi_{\infty}(t)-\xi_{+}(t)\right| & \leq\left|e^{-i\left(\Theta_{\infty}(t)+\Theta_{0}\right)}-e^{-i \Theta(t)}\right|\left|z_{+}\right| \\
& \leq\left|\left(\Theta_{\infty}(t)+\Theta_{0}\right)-\Theta(t)\right|\left|z_{+}\right| \leq C \varepsilon^{3}\langle\sigma\rangle^{-2 \nu-\kappa-\mu} t^{-\rho+\mu+1},
\end{aligned}
$$

which, together with (5.16), yields

$$
\left|\xi_{\infty}(t)-\xi(t)\right| \leq C \varepsilon\langle\sigma\rangle^{-\kappa-\mu} t^{-\rho+\mu+1}, \quad t \geq t_{0} .
$$

Observing that $\left|\xi_{\infty}(t)\right|=\left|z_{+}\right|$by definition, we find that

$$
\begin{aligned}
\xi_{\infty}^{\prime}(t) & =-i \Theta_{\infty}^{\prime}(t) \xi_{\infty}(t)=-i \frac{\operatorname{Im} K}{2 t \eta_{\infty}(t)}\left|\xi_{\infty}(t)\right|^{2} \xi_{\infty}(t), \\
\eta_{\infty}^{\prime}(t) & =\frac{\operatorname{Re} K}{t}\left|\xi_{\infty}(t)\right|^{2}
\end{aligned}
$$

for $t>1$. Hence if we put $z_{\infty}(t)=\xi_{\infty}(t) / \sqrt{\eta_{\infty}(t)}$, then we get

$$
z_{\infty}^{\prime}(t)=-\frac{K}{2 t}\left|z_{\infty}(t)\right|^{2} z_{\infty}(t), \quad t>1
$$


It follows from (5.15), (5.17), (5.19), (5.21), and (5.24) that

$$
\begin{aligned}
\left|z(t)-z_{\infty}(t)\right| & \leq \frac{\left|\xi(t)-\xi_{\infty}(t)\right|}{\sqrt{\eta(t)}}+\left|z_{+}\right|\left|\frac{1}{\sqrt{\eta(t)}}-\frac{1}{\sqrt{\eta_{\infty}(t)}}\right| \\
& \leq C \varepsilon\langle\sigma\rangle^{-\kappa-\mu} t^{-\rho+\mu+1}, \quad t \geq t_{0} .
\end{aligned}
$$

Finally we put $p_{0}:=z_{\infty}(1)$, and let $p$ be the solution to (5.7). It is clear that $z_{\infty}(t)=p(\log t)$ for $t \geq 1$. By (5.17) and (5.21), we get

$$
\left|p_{0}\right|=\left|\frac{\xi_{\infty}(1)}{\sqrt{\eta_{\infty}(1)}}\right| \leq C \varepsilon\langle\sigma\rangle^{-\nu} .
$$

We obtain (5.5) and (5.6) from (5.25) and (5.26). This completes the proof.

\section{Proof of Theorem 2.1}

In this section we prove Theorem 2.1. Let $u$ be a smooth solution to (1.1)(1.2) on $[0, T) \times \mathbb{R}^{2}$ for some $T>0$. For a positive integer $k$, and positive constants $\lambda$ and $\mu$, we define

$$
\begin{aligned}
e_{k, \lambda, \mu}[u](T):=\sup _{(t, x) \in[0, T) \times \mathbb{R}^{2}}\{ & (1+t)^{1 / 2}\langle t-r\rangle^{1-\mu}|\partial u(t, x)| \\
& \left.+(1+t)^{(1-\lambda) / 2}\langle t-r\rangle^{1-\mu}|\partial u(t, x)|_{k}\right\},
\end{aligned}
$$

where $r=|x|$. Our first aim here is to prove the following, from which the global existence part of Theorem 2.1 follows:

Proposition 6.1. Suppose that the assumptions in Theorem 2.1 are fulfilled. Let $k \geq 2$ and $0<\mu<1 / 10$. If $0<2 k \lambda \leq \mu / 6$, then we can find a positive constant $m_{0}=m_{0}(k, \lambda, \mu)$ having the following property: For any $m \geq m_{0}$ there is a positive constant $\varepsilon_{0}=\varepsilon_{0}(m, k, \lambda, \mu)$ such that

$$
e_{k, \lambda, \mu}[u](T) \leq m \varepsilon
$$

implies

provided that $0<\varepsilon \leq \varepsilon_{0}$.

$$
e_{k, \lambda, \mu}[u](T) \leq \frac{m}{2} \varepsilon
$$

Proof. Assume that (6.1) is satisfied. In the following we always suppose that $0 \leq t<T$. We also suppose that $m \geq 1$, and that $\varepsilon$ is small enough to satisfy $m \varepsilon \leq 1$. The letter $C$ in this proof stands for a positive constant which may depend on $k, \lambda$, and $\mu$, but are independent of $m, \varepsilon$, and $T$. The proof is divided into several steps.

Step 1: The energy estimates. For $l \leq 2 k$, it follows from (6.1) that

$$
\begin{aligned}
|F(\partial u)|_{l} & \leq C\left(|\partial u|^{2}|\partial u|_{l}+|\partial u|_{k}^{2}|\partial u|_{l-1}\right) \\
& \leq \frac{C^{*}}{2} m^{2} \varepsilon^{2}(1+t)^{-1}|\partial u|_{l}+C m^{2} \varepsilon^{2}(1+t)^{\lambda-1}|\partial u|_{l-1}
\end{aligned}
$$


with a positive constant $C^{*}=C^{*}(k, \lambda, \mu)$, where terms including $|\partial u|_{l-1}$ should be neglected if $l=0$.

From the energy inequality and (6.3) with $l=0$, we get

$$
\|\partial u(t)\|_{0} \leq C \varepsilon+C^{*} m^{2} \varepsilon^{2} \int_{0}^{t}(1+\tau)^{-1}\|\partial u(\tau)\|_{0} d \tau .
$$

Gronwall's lemma implies

$$
\|\partial u(t)\|_{0} \leq C \varepsilon(1+t)^{C^{*} m^{2} \varepsilon^{2}} .
$$

We are going to prove that there are positive constants $B_{l}=B_{l}(k, \lambda, \mu)$ for $0 \leq l \leq 2 k$ such that

$$
\|\partial u(t)\|_{l} \leq B_{l} \varepsilon(1+t)^{C^{*} m^{2} \varepsilon^{2}+\lambda l}
$$

for $0 \leq l \leq 2 k$. Indeed (6.5) for $l=0$ follows from (6.4). Suppose that (6.5) is true for some $0 \leq l \leq 2 k-1$. Then, by (6.3) we get

$$
\begin{aligned}
\|\partial u(t)\|_{l+1} \leq & C \varepsilon+C^{*} m^{2} \varepsilon^{2} \int_{0}^{t}(1+\tau)^{-1}\|\partial u(\tau)\|_{l+1} d \tau \\
& +C B_{l} m^{2} \varepsilon^{3} \int_{0}^{t}(1+\tau)^{C^{*} m^{2} \varepsilon^{2}+\lambda(l+1)-1} d \tau .
\end{aligned}
$$

Then Gronwall's lemma yields

$$
\|\partial u(t)\|_{l+1} \leq C \varepsilon(1+t)^{C^{*} m^{2} \varepsilon^{2}}+C \frac{B_{l}}{\lambda(l+1)} m^{2} \varepsilon^{3}(1+t)^{C^{*} m^{2} \varepsilon^{2}+\lambda(l+1)},
$$

which inductively implies the desired result because $m \varepsilon \leq 1$.

Step 2: Decay estimates of generalized derivatives of higher order. We suppose that $\varepsilon$ is so small to satisfy $C^{*} m^{2} \varepsilon^{2} \leq \mu / 6$, where the constant $C^{*}$ is from (6.5). Then (6.5) for $l=2 k$ implies

$$
\|\partial u(t)\|_{2 k} \leq C \varepsilon(1+t)^{\mu / 3}
$$

because we have assumed $2 k \lambda \leq \mu / 6$. (6.6) yields

$$
\begin{aligned}
\int_{\mathbb{R}^{2}}|F(\partial u)(\tau, y)|_{2 k} d y & \leq C m \varepsilon(1+\tau)^{(\lambda-1) / 2}\|\partial u(\tau)\|_{2 k}^{2} \\
& \leq C m \varepsilon^{3}(1+\tau)^{\mu-(1 / 2)} \leq C \varepsilon(1+\tau)^{\mu-(1 / 2)},
\end{aligned}
$$

because $\lambda / 2<\mu / 3$. It is well known that we have $\left|u_{0}(t, x)\right|_{2 k-1} \leq C \varepsilon(1+t)^{-1 / 2}$ for the solution $u_{0}$ to $\square u_{0}=0$ with initial data $\left(u_{0}, \partial_{t} u_{0}\right)=(\varepsilon f, \varepsilon g)$ at $t=0$ (see [7] for instance). Hence, by Lemma 3.1] we get

$$
\begin{aligned}
(1+t)^{1 / 2}|u(t, x)|_{2 k-1} & \leq C \varepsilon+C \int_{0}^{t} \int_{\mathbb{R}^{2}} \frac{|F(\partial u)(\tau, y)|_{2 k}}{(1+\tau)^{1 / 2}} d y d \tau \\
& \leq C \varepsilon(1+t)^{\mu}
\end{aligned}
$$

for $(t, x) \in[0, T) \times \mathbb{R}^{2}$. From Lemma 3.4 we obtain

$$
|\partial u(t, x)|_{2 k-2} \leq C\langle t-r\rangle^{-1}|u(t, x)|_{2 k-1} \leq C \varepsilon(1+t)^{\mu-(1 / 2)}\langle t-r\rangle^{-1}
$$


for $(t, x) \in[0, T) \times \mathbb{R}^{2}$.

Suppose that $R$ is a positive number to satisfy (4.1), so that we have (4.2). Recall the definition (4.5) of $\Lambda_{T, R}$. We put $\Lambda_{T, R}^{\mathrm{c}}:=\left([0, T) \times \mathbb{R}^{2}\right) \backslash \Lambda_{T, R}$.

If we have either $t / 2<1$ or $r<t / 2$, then we get

$$
\langle t-r\rangle^{-1} \leq C\langle t+r\rangle^{-1} \text {. }
$$

If $r>t+R$, then we have $u(t, x)=0$ by (4.2). Hence (6.8) implies

$$
\sup _{(t, x) \in \Lambda_{T, R}^{c}}(1+t)^{1 / 2}\langle t-r\rangle^{1-\mu}|\partial u(t, x)|_{2 k-2} \leq C \varepsilon \text {. }
$$

Step 3: Decay estimates for generalized derivatives of lower order. We suppose that $(t, x)=(t, r \omega) \in \Lambda_{T, R}$ throughout this step. Recall that $t^{-1}$, $r^{-1},(1+t)^{-1}$, and $\langle t+r\rangle^{-1}$ are equivalent to each other. We define $U, U^{(\alpha)}$, $H$, and $H_{\alpha}$ as in Section 4 .

By (6.7) and (6.8), we get

$$
|u(t, x)|_{\sharp, 2 k-2} \leq C \varepsilon t^{\mu-(1 / 2)}\langle t-r\rangle^{-1},
$$

where $|u(t, x)|_{\sharp, 2 k-2}$ is defined by (4.6). Hence we obtain from (4.7) that

$$
\sum_{|\alpha| \leq 2 k-2}\left|U^{(\alpha)}(t, x)\right| \leq C \varepsilon t^{\mu}\langle t-r\rangle^{-1} .
$$

If $t / 2=r$ or $t / 2=1$, then we have $t^{\mu} \leq C\langle t-r\rangle^{\mu}$, and we obtain

$$
\sum_{|\alpha| \leq 2 k-2}\left|U^{(\alpha)}(t, x)\right| \leq C \varepsilon\langle t-r\rangle^{\mu-1} \text { when either } t / 2=r \text { or } t / 2=1 .
$$

By Corollary 3.3 and (6.7), we get

$$
t^{1 / 2}|\partial u|_{l} \leq C \sum_{|\alpha| \leq l}\left|r^{1 / 2} \partial \Gamma^{\alpha} u\right| \leq C \sum_{|\alpha| \leq l}\left|U^{(\alpha)}\right|+C \varepsilon t^{\mu-1}, \quad l \leq 2 k-2 .
$$

Recall that $0<\mu<1 / 10$. By (6.7), (6.10), and Proposition 4.1 we get

$$
|H(t, x)| \leq C\left(\varepsilon^{3} t^{3 \mu-2}\langle t-r\rangle^{-2}+\varepsilon t^{\mu-2}\right) \leq C \varepsilon t^{3 \mu-2}\langle t-r\rangle^{-2 \mu} .
$$

We define $t_{0, \sigma}=\max \{2,-2 \sigma\}$ for $\sigma \leq R$. Note that the line segment $\{(t,(t+\sigma) \omega) ; 0 \leq t<T\}$, with $\sigma \leq R$ and $\omega \in \mathbb{S}^{1}$ being fixed, meets the boundary of $\Lambda_{T, R}$ at the point $\left(t_{0, \sigma},\left(t_{0, \sigma}+\sigma\right) \omega\right)$. We have

$$
(1+R)^{-1}\langle\sigma\rangle \leq t_{0, \sigma} \leq 2(1+|\sigma|) \leq 2 \sqrt{2}\langle\sigma\rangle, \quad \sigma \leq R .
$$

We define

$$
V_{\sigma, \omega}(t)=U(t,(t+\sigma) \omega)
$$

for $0 \leq t \leq T, \sigma \leq R$, and $\omega \in \mathbb{S}^{1}$. Then (4.3) leads to

$$
V_{\sigma, \omega}^{\prime}(t)=-\frac{1}{2 t} F(\hat{\omega})\left|V_{\sigma, \omega}(t)\right|^{2} V_{\sigma, \omega}(t)+H(t,(t+\sigma) \omega)
$$

for $t_{0, \sigma} \leq t<T$. Note that by (6.11) we have

$$
\left|V_{\sigma, \omega}\left(t_{0, \sigma}\right)\right| \leq C \varepsilon\langle\sigma\rangle^{\mu-1} .
$$


for $\sigma \leq R$ and $\omega \in \mathbb{S}^{1}$. By (6.13), (6.14), and (6.16), we can apply Lemma 5.1 to (6.15) (with $\rho=2-3 \mu$, and $\kappa=2 \mu$ ): (5.4) implies that

$$
\left|V_{\sigma, \omega}(t)\right| \leq C \varepsilon\langle\sigma\rangle^{\mu-1}, \quad t \geq t_{0, \sigma}
$$

where $C$ is a constant independent of $\sigma$ and $\omega$ (note that $0<\mu<1 / 10<\rho-1$ and $\kappa+\rho-1=1-\mu)$. Now we get $|U(t, r \omega)|=\left|V_{r-t, \omega}(t)\right| \leq C \varepsilon\langle t-r\rangle^{\mu-1}$, and with the help of (6.12) for $l=0$ we obtain

$$
\sup _{(t, x) \in \Lambda_{T, R}}(1+t)^{1 / 2}\langle t-r\rangle^{1-\mu}|u(t, x)| \leq C \varepsilon .
$$

Let $|\alpha| \leq k$. For a nonnegative integer $s$, we set

$$
\mathcal{U}^{(s)}(t, x):=\sum_{|\alpha| \leq s}\left|U^{(\alpha)}(t, x)\right|
$$

By (6.12) we get

$$
|\partial u(t, x)|_{|\alpha|-1} \leq C\left(t^{-1 / 2} \mathcal{U}^{(|\alpha|-1)}(t, x)+\varepsilon t^{\mu-(3 / 2)}\right) .
$$

We obtain from (6.10), (6.19), and Proposition 4.1 that

$$
\begin{aligned}
\left|H_{\alpha}(t, x)\right| & \leq C\left(\varepsilon^{3} t^{3 \mu-2}\langle t-r\rangle^{-2}+\varepsilon t^{\mu-2}+\varepsilon^{3} t^{3 \mu-4}+t^{-1}\left(\mathcal{U}^{(|\alpha|-1)}(t, x)\right)^{3}\right) \\
& \leq C \varepsilon t^{3 \mu-2}\langle t-r\rangle^{-2 \mu}+C t^{-1}\left(\mathcal{U}^{(|\alpha|-1)}(t, x)\right)^{3}
\end{aligned}
$$

We put

$$
V_{\sigma, \omega}^{(\alpha)}(t)=U^{(\alpha)}(t,(t+\sigma) \omega)
$$

for $0 \leq t<T, \sigma \leq R$, and $\omega \in \mathbb{S}^{1}$. From (4.4) we get

$$
\begin{aligned}
\left(V_{\sigma, \omega}^{(\alpha)}\right)^{\prime}(t)= & -\frac{F(\hat{\omega})}{2 t}\left(2\left|V_{\sigma, \omega}(t)\right|^{2} V_{\sigma, \omega}^{(\alpha)}(t)+\left(V_{\sigma, \omega}(t)\right)^{2} \overline{V_{\sigma, \omega}^{(\alpha)}(t)}\right) \\
& +H_{\alpha}(t,(t+\sigma) \omega)
\end{aligned}
$$

for $t_{0, \sigma} \leq t<T$. Hence by (6.16) and (6.20) we obtain

$$
\begin{aligned}
\frac{d}{d t}\left|V_{\sigma, \omega}^{(\alpha)}(t)\right|^{2}= & -\operatorname{Re}\left(\frac{F(\hat{\omega})}{t}\left(2\left|V_{\sigma, \omega}(t)\right|^{2}\left|V_{\sigma, \omega}^{(\alpha)}(t)\right|^{2}+\left(V_{\sigma, \omega}(t)\right)^{2}\left(\overline{V_{\sigma, \omega}^{(\alpha)}(t)}\right)^{2}\right)\right) \\
& +2 \operatorname{Re}\left(H_{\alpha}(t,(t+\sigma) \omega) \overline{V_{\sigma, \omega}^{(\alpha)}}(t)\right) \\
\leq & \frac{2 C_{*} \varepsilon^{2}}{t}\left|V_{\sigma, \omega}^{(\alpha)}(t)\right|^{2}+C\left(\varepsilon t^{3 \mu-2}\langle\sigma\rangle^{-2 \mu}+t^{-1}\left(\mathcal{V}_{\sigma, \omega}^{(|\alpha|-1)}(t)\right)^{3}\right)\left|V_{\sigma, \omega}^{(\alpha)}(t)\right|
\end{aligned}
$$


for $t_{0, \sigma} \leq t<T$, where $\mathcal{V}_{\sigma, \omega}^{(s)}(t):=\sum_{|\alpha| \leq s}\left|V_{\sigma, \omega}^{(\alpha)}(t)\right|$, and $C_{*}=C_{*}(k, \lambda, \mu)$ is a positive constant. Therefore it follows from (6.11) that

$$
\begin{aligned}
t^{-C_{*} \varepsilon^{2}}\left|V_{\sigma, \omega}^{(\alpha)}(t)\right| \leq & t_{0, \sigma}^{-C_{*} \varepsilon^{2}}\left|V_{\sigma, \omega}^{(\alpha)}\left(t_{0, \sigma}\right)\right|+C \varepsilon\langle\sigma\rangle^{-2 \mu} \int_{t_{0, \sigma}}^{t} \tau^{-C_{*} \varepsilon^{2}+3 \mu-2} d \tau \\
& +C \int_{t_{0, \sigma}}^{t} \tau^{-C_{*} \varepsilon^{2}-1}\left(\mathcal{V}_{\sigma, \omega}^{(|\alpha|-1)}(\tau)\right)^{3} d \tau \\
\leq & C \varepsilon\langle\sigma\rangle^{\mu-1-C_{*} \varepsilon^{2}}+C \int_{t_{0, \sigma}}^{t} \tau^{-C_{*} \varepsilon^{2}-1}\left(\mathcal{V}_{\sigma, \omega}^{(|\alpha|-1)}(\tau)\right)^{3} d \tau
\end{aligned}
$$

for $t_{0, \sigma} \leq t<T$, which leads to

$$
t^{-C_{*} \varepsilon^{2}} \mathcal{V}_{\sigma, \omega}^{(l)}(t) \leq C \varepsilon\langle\sigma\rangle^{\mu-1}+C \int_{t_{0, \sigma}}^{t} \tau^{-C_{*} \varepsilon^{2}-1}\left(\mathcal{V}_{\sigma, \omega}^{(l-1)}(\tau)\right)^{3} d \tau
$$

for $t_{0, \sigma} \leq t<T$ and $1 \leq l \leq k$. Using this inequality, we are going to prove that

$$
\mathcal{V}_{\sigma, \omega}^{(l)}(t) \leq \widetilde{B}_{l} \varepsilon t^{3^{l-1} C_{*} \varepsilon^{2}}\langle\sigma\rangle^{\mu-1}
$$

for $t_{0, \sigma} \leq t<T$ and $1 \leq l \leq k$ with some positive constant $\widetilde{B}_{l}=\widetilde{B}_{l}(k, \lambda, \mu)$. By (6.17) we have $\mathcal{V}_{\sigma, \omega}^{(0)}(t) \leq \widetilde{B}_{0} \varepsilon\langle\sigma\rangle^{\mu-1}$ with a positive constant $\widetilde{B}_{0}=\widetilde{B}_{0}(k, \lambda, \mu)$. Hence we get

$$
t^{-C_{*} \varepsilon^{2}} \mathcal{V}_{\sigma, \omega}^{(1)}(t) \leq C \varepsilon\langle\sigma\rangle^{\mu-1}+C \widetilde{B}_{0}^{3} \varepsilon^{3}\langle\sigma\rangle^{3 \mu-3} \int_{2}^{\infty} \tau^{-C_{*} \varepsilon^{2}-1} d \tau \leq \widetilde{B}_{1} \varepsilon\langle\sigma\rangle^{\mu-1}
$$

with $\widetilde{B}_{1}=C+C C_{*}^{-1} \widetilde{B}_{0}^{3}$, which leads to

$$
\mathcal{V}_{\sigma, \omega}^{(1)}(t) \leq \widetilde{B}_{1} \varepsilon\langle\sigma\rangle^{\mu-1} t^{C_{*} \varepsilon^{2}},
$$

and (6.21) for $l=1$ is shown. Next, suppose that (6.21) is true for some $l$ with $1 \leq l \leq k-1$. Then we get

$$
\begin{aligned}
t^{-C_{*} \varepsilon^{2}} \mathcal{V}_{\sigma, \omega}^{(l+1)}(t) & \leq C \varepsilon\langle\sigma\rangle^{\mu-1}+C \widetilde{B}_{l}^{3} \varepsilon^{3}\langle\sigma\rangle^{3 \mu-3} \int_{2}^{t} \tau^{\left(3^{l}-1\right) C_{*} \varepsilon^{2}-1} d \tau \\
& \leq \widetilde{B}_{l+1} \varepsilon\langle\sigma\rangle^{\mu-1} t^{\left(3^{l}-1\right) C_{*} \varepsilon^{2}}
\end{aligned}
$$

with $\widetilde{B}_{l+1}=C+C C_{*}^{-1}\left(3^{l}-1\right)^{-1} \widetilde{B}_{l}^{3}$, and we obtain

$$
\mathcal{V}_{\sigma, \omega}^{(l+1)}(t) \leq \widetilde{B}_{l+1} \varepsilon\langle\sigma\rangle^{\mu-1} t^{3^{l} C_{*} \varepsilon^{2}},
$$

which is (6.21) with $l$ replaced by $l+1$. Now (6.21) for $1 \leq l \leq k$ is established.

By (6.21), we obtain

$$
\sum_{|\alpha| \leq k}\left|U^{(\alpha)}(t, x)\right| \leq C \varepsilon(1+t)^{3^{k-1} C_{*} \varepsilon^{2}}\langle t-r\rangle^{\mu-1}, \quad(t, x) \in \Lambda_{T, R},
$$

which yields

$$
|\partial u(t, x)|_{k} \leq C \varepsilon(1+t)^{3^{k-1} C_{*} \varepsilon^{2}-(1 / 2)}\langle t-r\rangle^{\mu-1}, \quad(t, x) \in \Lambda_{T, R}
$$


with the help of (6.12). If we choose sufficiently small $\varepsilon$ to satisfy

$$
3^{k-1} C_{*} \varepsilon^{2} \leq \lambda / 2,
$$

it follows from (6.22) that

$$
\sup _{(t, x) \in \Lambda_{T, R}}(1+t)^{(1-\lambda) / 2}\langle t-r\rangle^{1-\mu}|\partial u(t, x)|_{k} \leq C \varepsilon .
$$

The final step. By (6.9), (6.18), and (6.23), we see that there exist two positive constants $\varepsilon_{0}=\varepsilon_{0}(m, k, \lambda, \mu)$ and $C_{0}=C_{0}(k, \lambda, \mu)$ such that

$$
e_{k, \lambda, \mu}[u](T) \leq C_{0} \varepsilon
$$

for $0<\varepsilon \leq \varepsilon_{0}$ (note that we have $2 k-2 \geq k$ for $k \geq 2$ ). If $m \geq \max \left\{2 C_{0}, 1\right\}$, then (6.24) implies (6.2) immediately.

We are in a position to prove Theorem 2.1.

Proof of Theorem 2.1. Let the assumptions in Theorem 2.1 be fulfilled. Suppose that $u$ is a local solution to (1.1)-(1.2) on $[0, T) \times \mathbb{R}^{2}$ for some $T>0$. We fix $\lambda$ and $k$ as in Proposition 6.1. We also fix $m$ satisfying $m \geq m_{0}$ and

$$
\left.\sup _{x \in \mathbb{R}^{2}}\langle r\rangle^{1-\mu}\left(|\partial u(t, x)|+|\partial u(t, x)|_{k}\right)\right|_{t=0} \leq \frac{m}{2} \varepsilon, \quad \varepsilon>0,
$$

where $m_{0}=m_{0}(k, \lambda, \mu)$ is from Proposition 6.1. Let $\varepsilon_{0}=\varepsilon_{0}(m, k, \lambda, \mu)$ also be from Proposition 6.1. We put

$$
T_{*}=\sup \left\{t \in[0, T) ; e_{k, \lambda, \mu}(t) \leq m \varepsilon\right\} .
$$

By the choice of $m$, we have $T_{*}>0$. Moreover we get $T_{*}=T$ for $0<\varepsilon \leq \varepsilon_{0}$, because if $T_{*}<T$ then Proposition 6.1 implies $e_{k, \lambda, \mu}\left(T_{*}\right)<m \varepsilon / 2$ for $0<\varepsilon \leq$ $\varepsilon_{0}$, and we obtain from the continuity of $e_{k, \lambda, \mu}$ that $e_{k, \lambda, \mu}\left(t_{*}\right) \leq m \varepsilon$ for some $t_{*}>T_{*}$, which contradicts the definition of $T_{*}$. Therefore we see that $e_{k, \lambda, \mu}(t)$ cannot exceed $m \varepsilon$ as long as the solution $u$ exists. This a priori estimate and the local existence theorem implies the global existence of the solution $u$. We also see that (6.1) holds for some large $m$.

Now we turn our attention to the asymptotic behavior. Because we have (6.1), the estimates in the proof of Proposition 6.1 are valid. We go back to (6.15), and apply Lemma 5.1 with $z(t)=V_{\sigma, \omega}(t), K=F(\hat{\omega}), J(t)=$ $H(t,(t+\sigma) \omega), t_{0}=t_{0, \sigma}$ for each fixed $(\sigma, \omega) \in(-\infty, R] \times \mathbb{S}^{1}$ (note that we can take $\rho=2-3 \mu$ and $\kappa=2 \mu$ because of (6.13), (6.14), and (6.16) $)$. Then we see that there exists $P_{0}=P_{0}(\sigma, \omega)$ such that

$$
\begin{aligned}
& \left|V_{\sigma, \omega}(t)-P(\log t, \sigma, \omega)\right| \leq C \varepsilon t^{4 \mu-1}\langle\sigma\rangle^{-3 \mu}, \quad t \geq t_{0, \sigma}, \\
& \left|P_{0}(\sigma, \omega)\right| \leq C \varepsilon\langle\sigma\rangle^{\mu-1},
\end{aligned}
$$

where $P=P(\tau, \sigma, \omega)$ is the solution to (2.5), and $C$ is a constant independent of $\sigma$ and $\omega$. Recalling that $D_{-}\left(r^{1 / 2} u(t, x)\right)=U(t, x)=V_{r-t, \omega}(t)$ with $r=|x|$ and $\omega=|x|^{-1} x$, we obtain from (6.25) that

$$
\left|D_{-}\left(r^{1 / 2} u(t, x)\right)-P(\log t, r-t, \omega)\right| \leq C \varepsilon t^{4 \mu-1}\langle t-r\rangle^{-3 \mu}
$$


for $(t, x) \in \Lambda_{T, R}$. By Corollary 3.3 and (6.7) we get

$$
\left|r^{1 / 2} \partial u(t, x)-\hat{\omega}(x) D_{-}\left(r^{1 / 2} u(t, x)\right)\right| \leq C \varepsilon(1+t)^{\mu-1},
$$

where $\hat{\omega}(x)=(-1, x /|x|)$. Since (6.26) and (2.8) yield $|P(\tau, \sigma, \omega)| \leq C \varepsilon\langle\sigma\rangle^{\mu-1}$, we have

$$
\begin{aligned}
\left|\left(r^{-1 / 2}-t^{-1 / 2}\right) P(\log t, r-t, \omega)\right| & \leq C \varepsilon \frac{|t-r|}{\sqrt{t} \sqrt{r}(\sqrt{t}+\sqrt{r})}\langle t-r\rangle^{\mu-1} \\
& \leq C \varepsilon t^{-3 / 2}\langle t-r\rangle^{\mu}
\end{aligned}
$$

for $(t, x) \in \Lambda_{T, R}$.

To sum up the estimates (6.27), (6.28) and (6.29), we arrive at

$$
\left|\partial u(t, x)-\hat{\omega}(x) t^{-1 / 2} P(\log t, r-t, \omega)\right| \leq C \varepsilon t^{4 \mu-(3 / 2)}\langle t-r\rangle^{-3 \mu}
$$

for $(t, x) \in \Lambda_{T, R}$. In order to extend (6.30) outside of $\Lambda_{T, R}$, we just have to extend the definition of $P_{0}$ by setting $P_{0}(\sigma, \omega)=0$ for $\sigma>R$. Indeed, both $u(t, x)$ and $P(\log t, r-t, \omega)$ vanish for $|x| \geq t+R$. On the other hand, if $r<t / 2$ or $1<t<2$, we have

$$
|\partial u(t, x)| \leq C \varepsilon t^{-1 / 2}\langle t-r\rangle^{\mu-1} \leq C \varepsilon t^{\mu-(3 / 2)},
$$

and

$$
t^{-1 / 2}|P(\log t, r-t, \omega)| \leq C \varepsilon t^{-1 / 2}\langle t-r\rangle^{\mu-1} \leq C \varepsilon t^{\mu-(3 / 2)} .
$$

Therefore (6.30) is valid for all $(t, x) \in[1, \infty) \times \mathbb{R}^{2}$, which shows (2.4). This completes the proof.

\section{Proof of Corollary 2.2}

Let the assumptions of Corollary 2.2 be fulfilled. Suppose that we have (4.1) for some $R>0$. Then we get (4.2). We always assume $t \geq 2$ throughout this proof. Since we have $R+|t-r| \leq \sqrt{2}(1+R)\langle t-r\rangle$, (2.10) leads to

$$
|\partial u(t, x)| \leq C t^{-1 / 2} \min \left\{(\log t)^{-1 / 2}, \varepsilon(R+|t-r|)^{\mu-1}\right\},
$$

where $r=|x|$.

We put $m_{\varepsilon}(t):=\varepsilon^{1 /(1-\mu)}(\log t)^{1 /(2-2 \mu)}$. Here $m_{\varepsilon}(t)$ is chosen so that we have $\varepsilon(R+t-r)^{\mu-1}=\varepsilon m_{\varepsilon}(t)^{\mu-1}=(\log t)^{-1 / 2}$ for $r=t+R-m_{\varepsilon}(t)$. For small $\varepsilon>0$ we have $0<m_{\varepsilon}(t)<t$, and we get $0<t+R-m_{\varepsilon}(t) \leq t+R$. Then it follows from (4.2) that

$$
\|u(t)\|_{E}^{2}=\frac{1}{2} \int_{|x| \leq t+R}|\partial u(t, x)|^{2} d x=I_{1}+I_{2}
$$

where

$$
I_{1}=\frac{1}{2} \int_{|x| \leq t+R-m_{\varepsilon}(t)}|\partial u(t, x)|^{2} d x, \quad I_{2}=\frac{1}{2} \int_{t+R-m_{\varepsilon}(t) \leq|x| \leq t+R}|\partial u(t, x)|^{2} d x .
$$


Note that we have $t^{-1} r \leq t^{-1}(t+R) \leq 1+R / 2$ for $0 \leq r \leq t+R$. Hence, switching to the polar coordinate, we get from (7.1) that

$$
\begin{aligned}
I_{1} & \leq C \varepsilon^{2} \int_{0}^{t+R-m_{\varepsilon}(t)} t^{-1}(R+|t-r|)^{2 \mu-2} r d r \\
& \leq C \varepsilon^{2} \int_{0}^{t+R-m_{\varepsilon}(t)}(R+t-r)^{2 \mu-2} d r \leq C \varepsilon^{2} m_{\varepsilon}(t)^{2 \mu-1}=C \varepsilon^{\frac{1}{1-\mu}}(\log t)^{-\frac{1-2 \mu}{2-2 \mu}} .
\end{aligned}
$$

Similarly it follows from (7.1) that

$$
\begin{aligned}
I_{2} & \leq C \int_{t+R-m_{\varepsilon}(t)}^{t+R} t^{-1}(\log t)^{-1} r d r \\
& \leq C(\log t)^{-1} \int_{t+R-m_{\varepsilon}(t)}^{t+R} d r=C \varepsilon^{\frac{1}{1-\mu}}(\log t)^{-\frac{1-2 \mu}{2-2 \mu}} .
\end{aligned}
$$

This completes the proof.

\section{ACKNOWLEDGMENTS}

The first author (S. K.) is supported by Grant-in-Aid for Scientific Research (C) (No. 23540241), JSPS. The third author (H. S.) is supported by Grant-inAid for Young Scientists (B) (No. 22740089), MEXT.

\section{REFERENCES}

[1] S. Alinhac, The null condition for quasi linear wave equations in two space dimensions I, Invent. Math. 145 (2001), 597-618.

[2] S. Alinhac, The null condition for quasi linear wave equations in two space dimensions II, Amer. J. Math. 123 (2001), 1071-1101.

[3] D. Christodoulou, Global solutions of nonlinear hyperbolic equations for small initial data, Comm. Pure Appl. Math. 39 (1986), 267-282.

[4] P. Godin, Lifespan of solutions of semilinear wave equations in two space dimensions, Comm. Partial Differential Equations, 18 (1993), 895-916.

[5] N. Hayashi, P. Naumkin and H. Sunagawa, On the Schrödinger equation with dissipative nonlinearities of derivative type, SIAM J. Math. Anal. 40 (2008), 278-291.

[6] L. Hörmander, $L^{1}, L^{\infty}$ estimates for the wave operator, in "Analyse Mathématique et Applications, Contributions en l'Honneur de J. L. Lions", Gauthier-Villars, Paris, 1988, 211-234.

[7] L. Hörmander, "Lectures on Nonlinear Hyperbolic Differential Equations", Math. Appl. 26, Springer-Verlag, Berlin, 1997.

[8] A. Hoshiga, The initial value problems for quasi-linear wave equations in two space dimensions with small data, Adv. Math. Sci. Appl. 5 (1995), 67-89.

[9] A. Hoshiga, The existence of the global solutions to semilinear wave equations with a class of cubic nonlinearities in 2-dimensional space, Hokkaido Math. J. 37 (2008), 669-688.

[10] S. Katayama, Global existence for systems of nonlinear wave equations in two space dimensions, Publ. RIMS, Kyoto Univ. 29 (1993), 1021-1041.

[11] S. Katayama, Global existence for systems of nonlinear wave equations in two space dimensions, II, Publ. RIMS, Kyoto Univ. 31 (1995), 645-665.

[12] S. Katayama, Asymptotic pointwise behavior for systems of semilinear wave equations in three space dimensions, to appear in J. Hyperbolic Differ. Eq. 
[13] S. Katayama, Asymptotic behavior for systems of nonlinear wave equations with multiple propagation speeds in three space dimensions, preprint.

[14] S. Klainerman, The null condition and global existence to nonlinear wave equations, in "Nonlinear Systems of Partial Differential Equations in Applied Mathematics, Part 1", Lectures in Applied Math. 23, Amer. Math. Soc., Providence, RI, 1986, 293-326.

[15] H. Kubo, Asymptotic behavior of solutions to semilinear wave equations with dissipative structure, Discrete Contin. Dynam. Systems, Supplement Volume 2007 (2007), 602-613.

[16] H. Lindblad, On the lifespan of solutions of nonlinear wave equations with small initial data, Comm. Pure Appl. Math. 43 (1990), 445-472.

[17] J. L. Lions and W. A. Strauss, Some nonlinear evolution equations, Bull. Soc. Math. France 93 (1965), 43-96.

[18] K. Mochizuki and T. Motai, On energy decay-nondecay problems for wave equations with nonlinear dissipative term in $\mathbb{R}^{N}$, J. Math. Soc. Japan 47 (1995), 405-421.

[19] D. Murotani, Large time behavior of solutions for a class of semilinear wave equations in 2D, Master Thesis, Osaka University, 2011.

[20] H. Sunagawa, Large time behavior of solutions to the Klein-Gordon equation with nonlinear dissipative terms, J. Math. Soc. Japan 58 (2006), 379-400.

[21] G. Todorova and B. Yordanov, The energy decay problem for wave equations with nonlinear dissipative terms in $\mathbb{R}^{n}$, Indiana Univ. Math. J. 56 (2007), 389-416.

Department of Mathematics, WAKayama UniVERsity, 930 SAKaEdani, Wakayama 640-8510, JAPAN

E-mail address: katayama@center.wakayama-u.ac.jp

Department of Mathematics, Graduate School of Science, Osaka UniverSITY, TOYONAKA, OSAKA 560-0043, JAPAN.

E-mail address: d-murotani@cr.math.sci.osaka-u.ac.jp

Department of Mathematics, Graduate School of Science, Osaka UniverSITY, TOYONAKA, OSAKA 560-0043, JAPAN.

E-mail address: sunagawa@math.sci.osaka-u.ac.jp 